\title{
Precise Indoor Localization using PHY Layer Information
}

\author{
Souvik Sen \\ Duke University \\ Romit Roy Choudhury \\ Duke University
}

\author{
Bozidar Radunovic \\ Microsoft Research, UK \\ Tom Minka \\ Microsoft Research, UK
}

\begin{abstract}
This paper shows the viability of precise indoor localization using physical layer information in WiFi systems. We find that channel frequency responses across multiple OFDM subcarriers can be suitably aggregated into a location fingerprint. While these fingerprints vary over time and environmental mobility, we notice that their core structure preserves certain properties that are amenable to localization. We demonstrate these ideas through a functional prototype, implemented on off-the-shelf Intel 5300 cards (that export per-subcarrier information to the driver). We evaluate the prototype using the existing APs inside a busy building, a cafeteria, and a museum, and demonstrate localization accuracies in the granularity of $1 \mathrm{~m} \times 1 \mathrm{~m}$ boxes, called spots. Results show that our system, PinLoc, is able to localize users to a spot with $90 \%$ mean accuracy, while incurring less than $6 \%$ false positives. We believe this holds promise towards an important development in indoor localization.
\end{abstract}

\section{Categories and Subject Descriptors}

C.2.1 [Network Architecture and Design]: Wireless communication

\section{General Terms}

Design, Experimentation, Performance

\section{Keywords}

Wireless, Localization, Cross-Layer, Application

\section{INTRODUCTION}

Precise indoor localization has been a long standing problem. While the frontier of localization technology has advanced over time, new kinds of location based applications are raising the bar. For instance, the advertising industry

Permission to make digital or hard copies of all or part of this work for personal or classroom use is granted without fee provided that copies are not made or distributed for profit or commercial advantage and that copies bear this notice and the full citation on the first page. To copy otherwise, to republish, to post on servers or to redistribute to lists, requires prior specific permission and/or a fee.

Hotnets '11, November 14-15, 2011, Cambridge, MA, USA.

Copyright 2011 ACM 978-1-4503-1059-8/11/11 ...\$10.00. is beginning to expect location accuracies at the granularity of an aisle in a grocery shop [1]. Museums are expecting user locations at the granularity of paintings [2] so users can automatically receive information about the paintings that they walk by. In addition to such high accuracy demands, these applications are inherently intolerant to small errors. If a localization scheme incorrectly places a user in the adjacent aisle in the grocery store, or downloads information about the adjacent painting, the purpose of localization is entirely defeated. This is unlike traditional applications - say GPS based driving directions - where small errors are tolerable. As a consequence, new localization schemes will need to meet strict standards, without incurring additional costs of installation and maintenance. We refer to this problem as spot localization, where a device in a specific $1 m \times 1 m$ box needs to be accurately identified. Localizing the device outside the box will be useless, irrespective of whether the estimated location is close or far away from the box.

The state of the art in indoor localization is quite sophisticated. Cricket [3] achieves high accuracy using special (ultrasound-based) infrastructure installed on ceilings. Noting the difficulties of installing special hardware, RADAR, Place Labs and Horus [4-6] explored the feasibility of using signal strengths from existing WiFi APs. While RADAR and Horus both rely on signal calibration, EZ [7] recently demonstrated the ability to eliminate calibration at the expense of accuracy degradation. Summarizing all these schemes, we find that the state of the art achieves median location error of $4 m$ and $7 m$, with and without calibration, respectively [7] . While this accuracy can enable a variety of applications, there are others that need precision at the granularity of " $1 \mathrm{~m} \times 1 \mathrm{~m}$ ". This paper targets such high accuracies while ensuring that the calibration complexity is no worse than RADAR or Horus. We call our proposal PinLoc, as an acronym for Precise indoor localization.

PinLoc's main idea is simple. While most WiFi based localization schemes operate with signal strength based information at the MAC layer, we recognize the possibility of leveraging detailed physical (PHY) layer information. Briefly, the intuition is that the multipath signal components ar- 
rive at a given location with distinct values of phase and magnitude. When carefully aggregated over multiple OFDM sub-carriers in $802.11 \mathrm{a} / \mathrm{g} / \mathrm{n}$, this rich data yield a fingerprint of that location. We find that, despite movements of people and other objects in the environment, devices are reliably classified to the correct spot. The war-driving effort is not significant (performed automatically by a Roomba robot), and may be similar to RADAR and Horus.

Our observations seemed coincidental at first glance - we expected the signal phases to be sensitive to the changes in environment, and learning them on a per-location basis seemed infeasible. However, upon continued investigation (discussed later), we observed that the phase and magnitude over 30 subcarriers richly capture the scattering in the environment. Moreover, dynamic obstructions in the environment distort the scattering in statistically reproducible ways, while some of the distortions are transient with mobile objects. Finally, even if some of the signal paths get permanently blocked, the diversity available from different APs injects resilience. All these factors together contribute towards a stable location fingerprint. RSSI, on the other hand, is an average of the magnitudes on each sub-carrier which hides fine-grained information about that location, ultimately limiting the accuracy of localization.

Translating the above high level ideas into a working prototype (using off-the-shelf wireless cards) forms the core of PinLoc. The detailed PHY layer information is first extracted from the driver and sanitized using phase correction operations. The sanitized parameters are then fed to a machine learning algorithm that models the channel response distribution. Later, during system tests, the channel parameters are extracted from received WiFi beacons, and classified to one of the war-driven spots. To address energy issues, PinLoc disables active scanning, and only uses beacons from APs in the same channel. Finally, the individual modules are combined into a full system, and tested over a range of scenarios. The results are promising, with $90 \%$ mean accuracy, and false positives below $6 \%$.

To the best of our knowledge, no prior work has demonstrated PHY layer-based WiFi localization on off-the-shelf platforms. Zhang et. al. [8] used signal amplitudes and phases on USRP platforms to demonstrate location distinction. We note that location distinction detects when a node's location has changed (e.g., for security purposes), but does not need to establish that each location exhibit uniqueness. Localization is naturally a far stricter problem, especially when the target is sub-meter accuracies.

\section{HYPOTHESES AND MEASUREMENTS}

This section aims to experimentally show that PHY layer channel information from existing WiFi deployments can be an indicator of location. The next section draws on the findings to design the components in PinLoc.

\subsection{Background}

Most modern digital radios use OFDM communication, and transmit signals across orthogonal subcarriers at different frequencies. Each transmitted symbol $X(f)$ is modulated on a different subcarrier $f$, and the quality of the received symbol $Y(f)$ will depend on the channel $H(f)$ :

$$
Y(f)=H(f) X(f)
$$

Vector $\mathbf{H}=(H(f))_{f=1, \cdots, F}$ is called channel frequency response (CFR), and it is a complex vector that describes the channel performance at each subcarrier. A $802.11 \mathrm{a} / \mathrm{g} / \mathrm{n}$ receiver implements $F=48$ data sub-carriers, and includes a channel estimation circuit as a part of the hardware. The Intel 5300 [9] card, released recently with a publicly downloadable driver, exposes the per-subcarrier CFR to the user. Figure $1(a, c)$ shows examples of some $C F R$ vectors.

Two important properties of the CFR are of interest in PinLoc. (1) The CFR changes entirely once a transmitter or a receiver moves more than a fraction of a wavelength $(12 \mathrm{~cm}$ in case of WiFi [10]). (2) Even if the device is static at a specific location, the CFR experiences channel fading due to changes in the environment at different time-scales. This perturbs the location signatures, injecting ambiguity in localization. However, it is unclear if these perturbations are completely random - if they are not, then the fingerprint may exhibit some structure, making them amenable to precise localization. The following measurements are designed to answer these related questions.

\subsection{Experiment Methodology}

Our initial experiments were performed in a relatively busy engineering building. We consider a set of 15 different spots, at approximately $2 m$ apart from each other, in our lab and the adjacent classroom. We place a laptop equipped with the Intel $5300 \mathrm{WiFi}$ card [9] at each of these spots, and associate them to existing WiFi APs. The laptops are made to download packets through each of the nearby APs - the corresponding channel frequency responses (CFR) are recorded for each packet. For each location we perform 4-6 measurements at different times of the day, during busy office hours, in the presence of several people.

\subsection{Measurement, Hypotheses and Verification}

With the above setup, we present and verify two main hypotheses that underpin PinLoc:

(Hypothesis 1) The CFRs at each location are not entirely random, and actually exhibit a defined structure across time.

Testing on a Single Location: Figure 1(a) shows the CFR recorded on a laptop at a fixed location (the laptop received 20,000 packets from a specific AP over a period of $100 s$, but we only show 50 randomly selected packets to avoid visual clutter). We observe two emerging clusters, denoted with two vectors $\mathbf{U}_{1}$ and $\mathbf{U}_{2}-$ CFRs belonging to the same clus- 

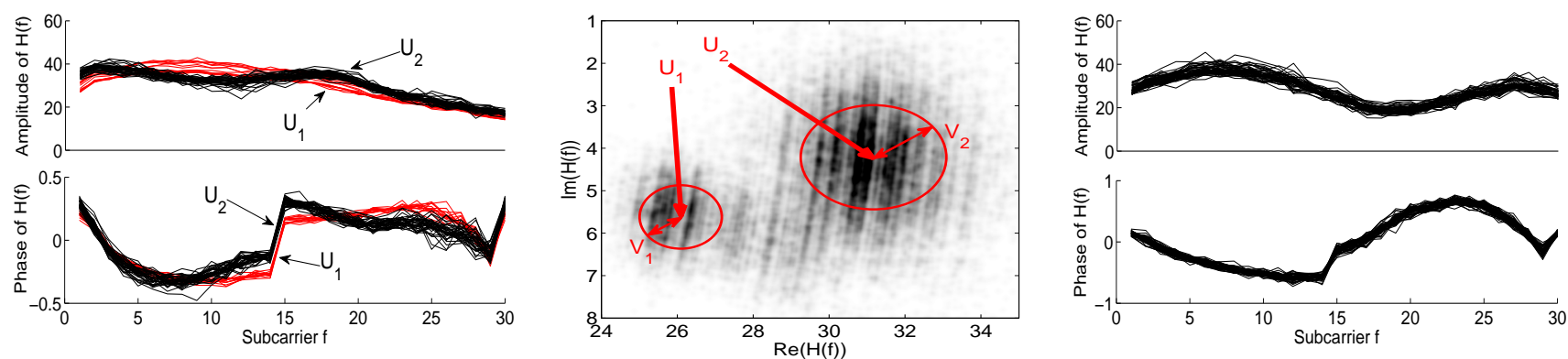

Figure 1: (a) The amplitudes and phases of the channel responses $\mathrm{H}$ of 50 (out of 20000) packets sent over the same link (we see two unique clusters, $\mathbf{U}_{1}$ and $\mathbf{U}_{2}$ ); (b) PDF of the complex value of the same 20000 channel responses $H(f)$ for a single subcarrier $f=20$; (c) The amplitudes and phases of the channel responses $\mathrm{H}$ of 50 packets at a different link.

ter are not identical but appear as noisy realizations of the cluster mean. This is an outcome of fast-fading, caused by different electro magnetic propagation effects.

Figure 1(b) plots the empirical probability density function (PDF) of the complex values of the CFR at the same location for a single subcarrier $f=20$. The PDF is constructed from all 20,000 packets. Darker colors represent higher values of the PDF. We again see that two dominant clusters emerge, each cluster appearing as a complex Gaussian random variable, with means $U_{1}(f)$ and $U_{2}(f)$ and variances $V_{1}(f)$ and $V_{2}(f)$ respectively. Of course, this is only a visual indication - we discuss a general clustering methodology later in $\S 3$. Figure 1(c) shows the outcome of the same experiment, but with the laptop placed at a different location. We find only a single cluster of CFRs, and the shape of the CFRs differs distinctly from those observed in Figure 1(a). These few representative clusters hint at the possible existence of complex but stable structures in per-location CFR, motivating further investigation.

Many Locations: We now test whether the observations from these two locations generalize to a larger number of locations, under various environmental changes. Figure 2 shows the distribution of the number of representative CFR clusters from 30 distinct links in total. For each of the 30 links we aggregate all the available data from different times of days, to get more complete information about the link's diversity. Evident from Figure 2 (a), more than $80 \%$ of cases experience 4 CFR clusters or less. However, we still see a substantial number of locations with a large number of clusters, even up to 19. This could well suggest that the CFR structure is quite random in dynamic scenarios (e.g., in the classroom), and thus, PinLoc may only be applicable in very static environments.

To verify this, we next look at frequency of occurrence of different clusters. Figure 2(b) shows that the distribution is highly non-uniform, with a strong predominance of the more frequent clusters (i.e., frequent clusters occur very frequently, and the vice versa). More precisely, the fourthmost frequent cluster occurs no more than $10 \%$ of the cases
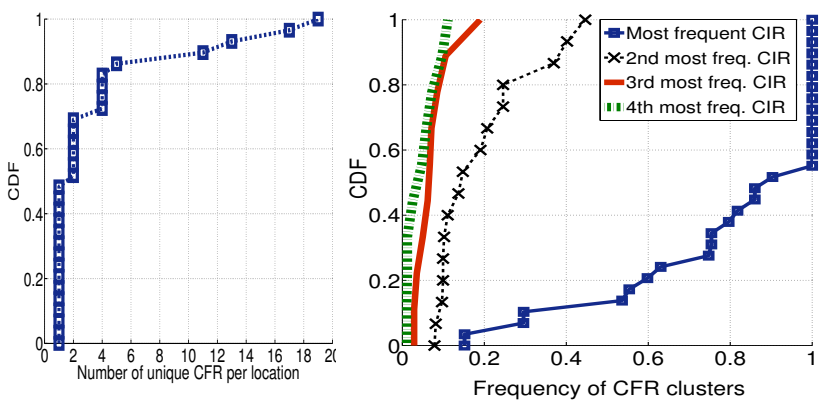

Figure 2: (a) CDF of the number of CFR clusters observed at different locations; (b) CDF of the probability of seeing the $n$-th most frequent CFR cluster.

in any spot, and the $5^{\text {th }}, 6^{\text {th }}, \ldots 19^{\text {th }}$ clusters are almost rare. This suggests that even if a few spots experience large number of clusters, we are not very likely to see most of them during the localization phase.

Impact of environmental changes: The above observation is surprising especially because the measurement was conducted in presence of many humans. Humans are typically the most dynamic obstacles in the environment, and are believed to disrupt WiFi signals. To shed more light on the impact of human mobility onto CFRs, we perform a controlled experiment. We place a laptop at a fixed location and gather CFRs from two different APs. We run the experiment during night, and observe a single CFR cluster for each link. Then, we position a human at an increasing distance $d$ from the laptop.

We compute the cross-correlation of each received CFR with the CFR cluster observed without the human - we plot the CDF of the computed values. Figure 3 shows that human obstructions do not create a significant change to the CFRs from AP6230, probably because the human does not alter any of the strong signal paths between the laptop and the AP. The link to AP5A10, however, changes with human movement; nevertheless, the change is substantial only when the human is very close to the laptop ( 1 foot away). \$4 reports results from a busy cafeteria, showing that PinLoc can be robust over time, even in presence of hundreds of moving users. 

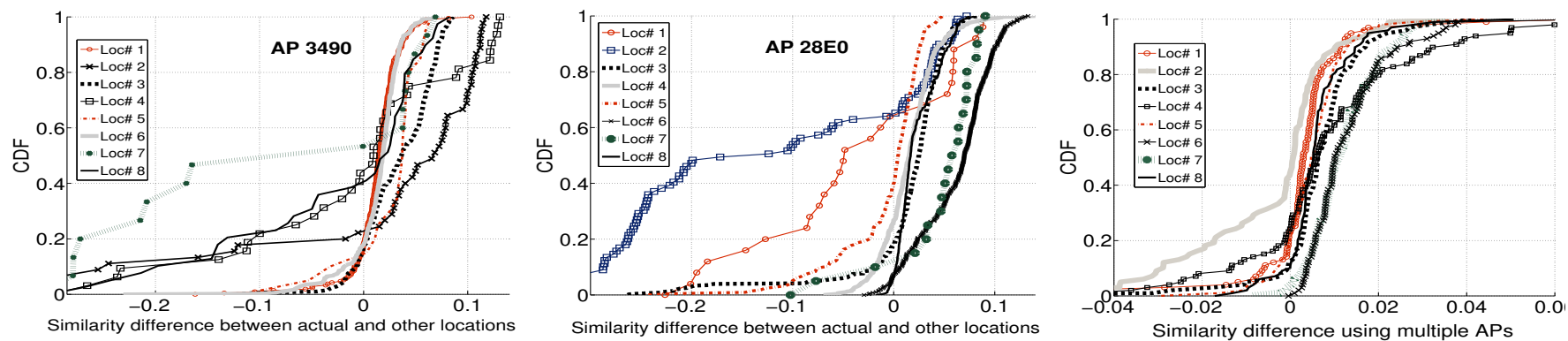

Figure 4: CDF of the difference in similarities $S_{\text {own }}-S_{\text {other }}$ observed at 8 locations, for two different access point: (a) AP 3490, (b) AP 28E0. (c) CDF of the maximum similarity difference $\left(S_{\text {own }}-S_{\text {other } s}\right)$ across all APs.
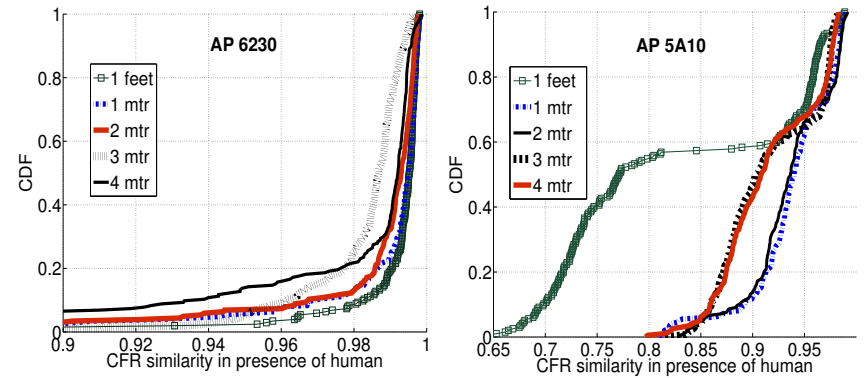

Figure 3: CFR cross correlation in presence of human beings at a location for 2 different APs

(Hypothesis 2) The CFR structure of a given location is different from the structures of all other locations.

To evaluate the (dis)similarities of CFRs among different locations, we divide the measured data into a training and a test set. Each location has a set of CFR clusters pertaining to an AP (represented by their mean and variance). For a test CFR from a link $L$, we use correlation to find the best matching CFR cluster from $L$ 's training data. The correlation value indicates similarity of the test CFR with its own link, denoted as $S_{\text {own }}$. Similarly, for all other links to the same AP (each link corresponding to a different location), we find the one that exhibits maximum similarity of this test CFR - we denote this similarity as $S_{\text {other }}$. If a device's measured CFR is more similar to a different link than its own link, we will naturally misclassify the device's location.

Figure 4 (a) and (b) plot the CDF of the difference in similarities $S_{\text {own }}-S_{\text {other }}$ for two different APs, for different locations. If the difference is negative, then the packet is likely to be misclassified. Also, the larger the difference, the greater the confidence in packet location.

Figure 4 (a) and (b) show that the CFR from a single AP is often sufficient to correctly classify location. Of course, in some cases - such as [Location7, AP3490] - more than $50 \%$ of the CFRs are more similar to other locations, implying misclassification. However, when considering the CFRs of location 7 to a different AP, the misclassification reduces significantly. This suggests that CFRs are diverse across different APs, and this diversity can be leveraged to improve localization. Figure 4 (c) shows the effect of exploiting AP diversity with 2 APs. Specifically, we now pick the AP with the highest similarity difference. Clearly, there is significantly less negative values in Figure 4 (c), implying greater location accuracy.

One may ask: Figure 4 shows that a packet may be classified to one out of 8 different spots. In reality, the system will need to discriminate between many more spots will the system scale to such scenarios? We note that PinLoc does not need to discriminate between all spots in a large area. Prior work has used WiFi SSIDs alone to localize devices to around $10 \mathrm{~m} \times 10 \mathrm{~m}$ regions in indoor environments [4]. PinLoc will leverage such schemes to first compute a coarse-grained macro-location, and then discriminate only between the spots inside that macro-location. Having verified these hypotheses, we present PinLoc's design. Thereafter, we evaluate PinLoc's performance in $\S 4$.

\section{SYSTEM DESIGN}

Learning: PinLoc measures the CFRs at spots of interest during the training phase and tries to identify as many of the unique clusters (observed in $\S 2$ ) as possible during a wardriving period. We make a reasonable assumption to model the noise (also called fast-fading) as a complex Gaussian noise, which corresponds to Rayleigh fading [10]. Consequently, we model the data at each location as a Gaussian mixture distribution [11] with $K$ clusters (we take $K=$ 10 in our case $)^{1}$. We estimate the parameters of the clusters (the mean and the variance of each cluster) using variational Bayesian inference [11]. We use the Infer.NET [12] framework to implement the clustering algorithm.

Classification: Our classification algorithm is composed of two parts. First, PinLoc computes a macro-location based on WiFi SSIDs alone [4], and shortlists the spots within this macro-location; we call these spots the candidate set, $C$. The second task is to pick one spot from $C$, or to declare that the device is not in any of these spots. To this end, the WiFi device overhears beacons from the APs as it roams around. We use log-likelihood [11] as a distance metric to express how likely is that the observed CFR belongs to a particular cluster from our training data-base. We find the

\footnotetext{
${ }^{1}$ Some locations may actually have more than 10 clusters but this is rare and discarding them has little impact on performance.
} 

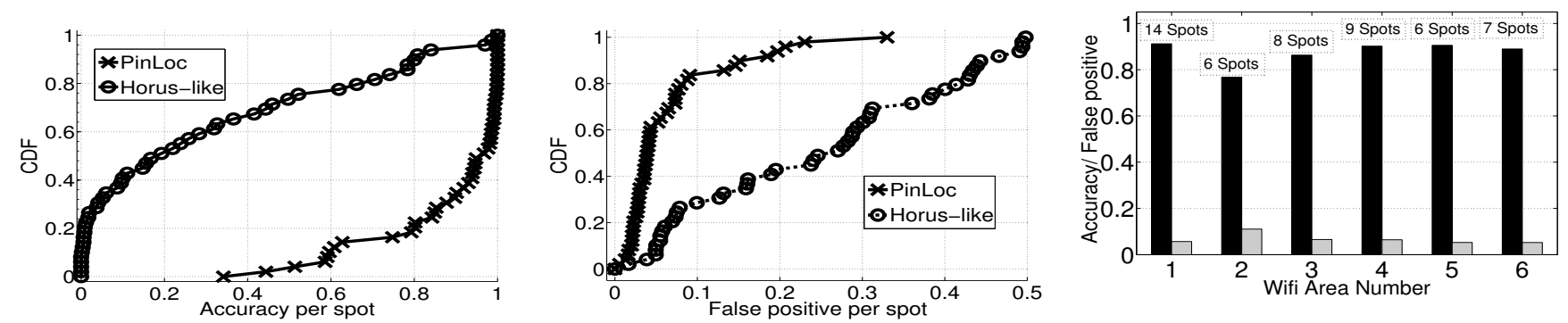

Figure 5: Pinloc performance in office environment (a) Accuracy, (b) False positive comparison with Horus (c) Per wifiarea performance.

best matching cluster from the data-base which indicates the most likely spot location. The operation repeats for every packet received within a short time window (typically 30 packets from $3 \mathrm{APs}$ ), and the spot that is picked most often (highest vote) is identified. PinLoc does not immediately declare the highest voted spot as the user's location. If the highest vote count is small (below a rejection threshold), it suggests low confidence and the possibility that the user is not located at any of the trained spots.

\section{EVALUATION}

War-driving and Experiment Design: We evaluate PinLoc across 66 different spots in 3 environments: (1) engineering building with offices and classrooms, (2) busy university cafeteria (3) the university museum. Each spot is $1 \mathrm{~m}$ $\times 1 \mathrm{~m}$ in size. We war-drive with a laptop mounted on a Roomba robot that moves in different directions across each spot (Fig. 6). Every spot is measured for 4 minutes to find the representative clusters in the training phase. Note that we do not need to learn all the clusters in a spot; it is enough to match one of the AP beacons to a learned cluster. However, more clusters we observe in the training and more AP beacons we match, its more likely we are to successfully localize $^{2}$. During the testing phase, the laptop associates to all APs on the same frequency channel, and receive beacon packets from them for a duration of $1 s$. This $1 s$ duration ensures that a mobile user (walking at $1 \mathrm{~m} / \mathrm{s}$ ) remain inside the same spot while receiving beacons. Test samples were collected on a different day, with more than 50 people around, along with a high churn. PinLoc attempts to correctly associate these test samples to a measured spot.

Metrics and Comparison: We use the two metrics to evaluate PinLoc. (1) Accuracy - the fraction of cases in which the user was localized to the correct spot. (2) False positives (FP) - the fraction of cases in which users were localized to an incorrect spot/non-spot. In other words, false positives also account for cases where PinLoc localizes the user to a trained spot, even though the user was not located at any of these spots. We also compare PinLoc with Horus to evaluate whether RSSI can achieve similar accuracy.

\footnotetext{
${ }^{2}$ Although smaller spots (upto $2 \mathrm{~cm} \times 2 \mathrm{~cm}$ ) could be evaluated, we limit the spot size to reduce war-driving complexity.
}

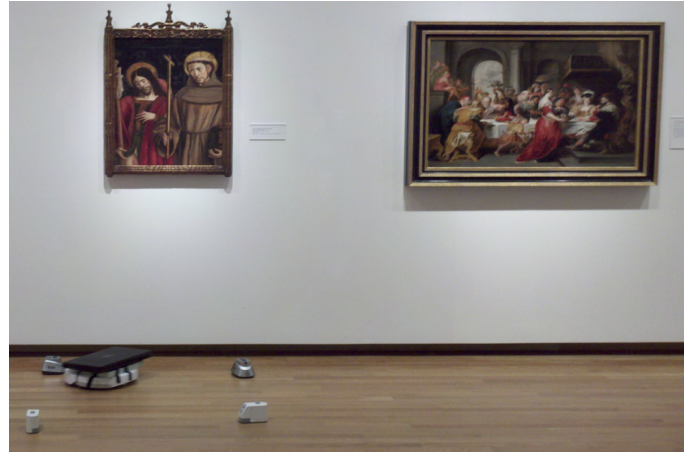

Figure 6: PinLoc war-driving at different spots in the museum. The Rhoomba robot mounted with a laptop, and 4 virtual wall devices at the corners of the spot.

\subsection{PinLoc accuracy and false positives}

Figure 5 reports results from the engineering building experiments. PinLoc achieves nearly $90 \%$ mean accuracy across 50 spots (Figure 5(a)), consistently outperforming Horus. False positives (FP) are also less than 7\%, compared to more than 25\% in Horus (Figure 5(b)). RSSI based algorithm is significantly worse than PinLoc, since it is represented with a single real number. CFR is represented with $30 \mathrm{com}$ plex numbers and contains much richer information.

Figure 5(c) zooms in to show the accuracy/FP on a perWiFi macro location. The number of spots per WiFi region is shown on top of the bars. Similar accuracy/FP graphs are plotted for cafeteria experiments in Figure 7(a). The mean accuracy for the cafeteria case is $90.07 \%$ and the mean FP is $4.5 \%$. We subject PinLoc to a real application scenario through experiments in the museum. PinLoc trains and localizes 10 large paintings in one wing of the museum (Figure 7 (b)). Mean accuracy of $90.28 \%$ and FP of $4.1 \%$ suggests that PinLoc can potentially enable painting-level localization. In all three scenarios, PinLoc achieves high accuracy for most of the spots, except around $20 \%$ where the performance drops. Careful investigation showed that these spots received packets at low SNR from many APs.

\section{Impact of mobility:}

We turn to the cafeteria scenario to analyze the effect of human mobility on localization accuracy. We take one hour of test data for three spots in the cafeteria. We localize each batch of 10 beacon packets and plot its success or failure in Figure 7 (c). We find that the time instants when localiza- 


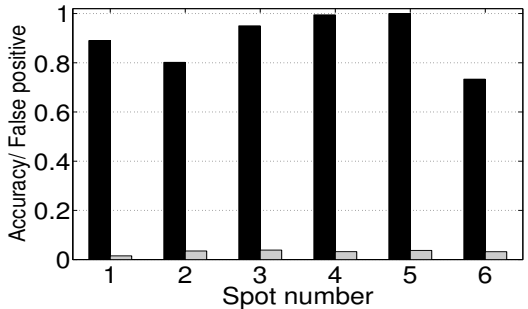

Figure 7: PinLoc performance at (a)

tion failed are short and uniformly spread over the measurement interval. The mean accuracy was $85 \%$ with $7 \%$ false positives. Thus, even in busy environments such as the cafeteria, PinLoc is able to provide robust localization.

\section{DISCUSSION AND FUTURE WORK}

- Height, orientation, phone mobility: PinLoc's war-driving and testing were performed on a 2D plane. In reality, since users carry the mobile phone at different heights, it is unclear whether war-driving on one horizontal plane will scale to a different plane. Issues may also emerge from phone orientation, or users inserting their phones in pockets, bags. Subjecting PinLoc to such real user scenarios will introduce new challenges requiring deeper investigation and understanding - we leave this investigation to future work.

- Structural changes in the environment: Our evaluation consists of environmental changes with respect to human mobility, and movement of chairs and objects. Major structural changes, such as repositioning of metal shelves and furniture, or changes in AP positions, are likely to "derail" PinLoc - a new round of war-driving would be necessary to cope with them. However, as long as these structural changes are in the order of large time scales (months or years), the overhead of war-driving may be well amortized.

\section{RELATED WORK}

The topic of indoor localization is well studied. RSSI-based localization such as RADAR [4], Horus [6] utilizes previously measured SSIDs and WiFi signal strength pattern for localization. Place Lab [5] and the Active Campus [13] reduce the measurement overhead; coupling information from WiFi and GSM base stations. Patwari et. al. [8] have recently explored the use of WiFi signal characteristics to identify when a device has moved from one location to another. They compare two observed channel responses and determine whether they belong to the same location. It does not attempt to extract features from responses and map them to locations, and hence it cannot be used for localization.

Time-based techniques such as PinPoint [14], and TPS [15] utilize signal propagation delay to estimate distances between wireless transmit-receiver pairs. The Cricket system $[3,16]$ utilize propagation delays between ultrasound and RF signals for localization; requiring special installation of ultrasound detectors. Recently angle-of-arrival based techniques utilize multiple antennas to estimate angles at which signals arrive, and then geometrically localize devices [17]. These techniques require extremely sophisticated antenna systems and non-trivial signal processing capabilities, unforeseeable on mobile devices in the near future. PinLoc's reliance on WiFi alone, along with the ability to utilize PHY layer information from off-the-shelf cards, makes it attractive for immediate deployment.

\section{CONCLUSIONS}

This paper shows that PHY layer information, exported by off-the-shelf Intel 5300 cards, can be adequate to localize WiFi devices to meter accuracies in indoor environments. We leverage the observation that multipath signals exhibit stable patterns, and these patterns can lead to precise localization. Evaluation results from the engineering building, cafeteria and university museum demonstrate a mean accuracy of $90 \%$ for 66 spots. We believe this is a step forward in the area of indoor localization, even though substantial more work is necessary for real-world deployment.

\section{Ri] placecast. Shopilerts.}

http://placecast.net/shopalerts/index.html.

[2] E. Bruns et al. Enabling mobile phones to support large-scale museum guidance. Multimedia, IEEE, 2007.

[3] N. B. Priyantha. The cricket indoor location system. $\mathrm{PhD}$ thesis.

[4] V. Bahl et al. RADAR: An in-building rf-based user location and tracking system. In INFOCOM, 2000.

[5] Yu-Chung et al. Accuracy characterization for metropolitan-scale wi-fi localization. In MobiSys, 2005.

[6] M. Youssef and A. Agrawala. The horus WLAN location determination system. In MobiSys, 2005.

[7] K. Chintalapudi, A. Iyer, and V. Padmanabhan. Indoor localization without the pain. In MOBICOM, 2010.

[8] Junxing Zhang et al. Advancing wireless link signatures for location distinction. In MobiCom. ACM, 2008.

[9] Intel Research. Intel 5300 mimo channel measurement tool. http://ils.intel-research.net/ 80211n-channel-measurement-tool.

[10] D. Tse and P. Viswanath. Fundamentals of Wireless Communication. Cambridge University Press, 2005.

[11] C. Bishop. Pattern Recognition and Machine Learning. Springer.

[12] T. Minka, J.M. Winn, J.P. Guiver, and D.A. Knowles. Infer.NET 2.4, 2010. Microsoft Research Cambridge.

[13] William G. Griswold et al. Activecampus: Experiments in community-oriented ubiquitous computing. Computer, 2004.

[14] M. Youssef et al. Pinpoint: An asynchronous time-based location determination system. In ACM Mobisys, June 2006.

[15] X. Cheng et al. TPS: A time-based positioning scheme for outdoor sensor networks. In IEEE Infocom, March 2004.

[16] A. Smith, Balakrishnan, et al. Tracking moving devices with the cricket location system. In ACM Mobisys, June 2004.

[17] J. Xiong and K. Jamieson. SecureAngle: improving wireless security using angle-of-arrival information. In ACM HotNets, 2010. 